\title{
Inclusive four-jet production: a study of Multi-Regge kinematics and BFKL observables
}

\section{Francesco Caporale}

Instituto de Física Teórica UAM/CSIC, Nicolás Cabrera 15, Madrid, Spain

Universidad Autónoma de Madrid, 28049 Madrid, Spain E-mail:

francesco.caporale@uam.es

\section{Francesco Giovanni Celiberto*}

Dipartimento di Fisica, Università della Calabria and Istituto Nazionale di Fisica Nucleare, Gruppo Collegato di Cosenza, Arcavacata di Rende, 87036 Cosenza, Italy, Instituto de Física Teórica UAM/CSIC, Nicolás Cabrera 15, Madrid, Spain Universidad Autónoma de Madrid, 28049 Madrid, Spain E-mail:

francescogiovanni.celibertodfis.unical.it

\section{Grigorios Chachamis}

Instituto de Física Teórica UAM/CSIC, Nicolás Cabrera 15, Madrid, Spain

Universidad Autónoma de Madrid, 28049 Madrid, Spain

chachamis@gmail.com

\section{Agustín Sabio Vera}

Instituto de Física Teórica UAM/CSIC, Nicolás Cabrera 15, Madrid, Spain

Universidad Autónoma de Madrid, 28049 Madrid, Spain E-mail:

a.sabio.vera@gmail.com

\begin{abstract}
A study of differential cross sections for the production of four jets in multi-Regge kinematics is presented, the main focus lying on azimuthal angle dependences. The theoretical setup consists in the jet production from a single BFKL ladder with a convolution of three BFKL Green functions, where two forward/backward jets are always tagged in the final state. Furthermore, the tagging of two further jets in more central regions of the detectors with a relative separation in rapidity from each other is requested. It is found, as result, that the dependence on the transverse momenta and the rapidities of the two central jets can be considered as a distinct signal of the onset of BFKL dynamics.
\end{abstract}

XXIV International Workshop on Deep-Inelastic Scattering and Related Subjects 11-15 April, 2016

DESY Hamburg, Germany

\footnotetext{
* Speaker.
} 


\section{Introduction}

The study of semi-hard processes in the high-energy (Regge) limit is an ultimate testfield for pQCD, the Large Hadron Collider (LHC) providing with an abundance of data. Multi-Regge kinematics (MRK), which prescribes a strong ordering in rapidity for the final state objects, is the key ingredient for the study of multi-jet production at LHC energies. In this kinematical regime, the common basis for the perturbative description of semi-hard processes is the Balitsky-FadinKuraev-Lipatov (BFKL) approach, at leading (LL) [1, 2, 3, 4, 5, 6] and next-to-leading (NLL) [7, 8] accuracy, which offers a resummation of those enhanced terms in MRK in regions of phase space where a fixed order calculation might not be enough. This formalism has been successfully applied to lepton-hadron Deep Inelastic Scattering at HERA (see, e.g. $[9,10]$ ) in order to study quite inclusive processes which are not that suitable though to discriminating between BFKL dynamics and other resummations. At the LHC, however, it is possible to investigate processes with much more exclusive final states which could, in principle, be only described by the BFKL framework, allowing us to disentangle the applicability region of the approach. In these last years, the most investigated process was the Mueller-Navelet jet production [11]. Interesting observables associated to this reaction are the azimuthal correlation momenta which, however, are strongly affected by collinear contaminations. Therefore, new observables independent from the conformal contribution were proposed in $[12,13]$ and calculated at NLL in $[14,15,16,17,18,19,20]$, showing a very good agreement with experimental data at the LHC. Nevertheless, Mueller-Navelet configurations are still too inclusive to perform MRK precision studies. For this reason a new study that demands the tagging of a third, central in rapidity, jet within the usual Mueller-Navelet configuration was proposed [21, 22]. Another interesting and novel possibility, the detection of two charged light hadrons: $\pi^{ \pm}, K^{ \pm}, p, \bar{p}$ having high transverse momenta and separated by a large interval of rapidity, together with an undetected soft-gluon radiaton emission, was suggested in [23] and studied in [24]. In the present work we follow the course taken in [21, 22], by allowing the production of a second central jet, which makes it possible to define new, generalized differential distributions [25] in the transverse momenta, azimuthal angles and rapidities of the two central jets, for fixed values of the four momenta of the forward jets.

\section{Inclusive four-jet production}

We study events with two forward/backward jets together with two more central jets, all of them well separated in rapidity from each other, making use of the BFKL formalism. The two tagged forward/backward jets $A$ and $B$ have transverse momentum $\vec{k}_{A, B}$, azimuthal angle $\vartheta_{A, B}$ and rapidity $Y_{A, B}$, while the pair of tagged more central jets are characterized, respectively, by $\vec{k}_{1,2}, \vartheta_{1,2}$ and $y_{1,2}$. The differential cross section is

$$
\begin{gathered}
\frac{d^{6} \sigma^{4-\text { jet }}\left(\overrightarrow{k_{A}}, \overrightarrow{k_{B}}, Y_{A}-Y_{B}\right)}{d^{2} \overrightarrow{k_{1}} d y_{1} d^{2} \overrightarrow{k_{2}} d y_{2}}=\frac{\bar{\alpha}_{s}^{2}}{\pi^{2} k_{1}^{2} k_{2}^{2}} \int d^{2} \overrightarrow{p_{A}} \int d^{2} \vec{p}_{B} \int d^{2} \vec{p}_{1} \int d^{2} \vec{p}_{2} \delta^{(2)}\left(\overrightarrow{p_{A}}+\overrightarrow{k_{1}}-\overrightarrow{p_{1}}\right) \\
\delta^{(2)}\left(\overrightarrow{p_{B}}-\overrightarrow{k_{2}}-\overrightarrow{p_{2}}\right) \varphi\left(\vec{k}_{A}, \vec{p}_{A}, Y_{A}-y_{1}\right) \varphi\left(\vec{p}_{1}, \vec{p}_{2}, y_{1}-y_{2}\right) \varphi\left(\overrightarrow{p_{B}}, \overrightarrow{k_{B}}, y_{2}-Y_{B}\right) .
\end{gathered}
$$

Here we have introduced the rapidity ordering characteristic of MRK: $Y_{A}>y_{1}>y_{2}>Y_{B}$; and $k_{1}^{2}$, $k_{2}^{2}$ lie above the experimental resolution scale. $\varphi$ are BFKL gluon Green functions normalized to 
$\varphi(\vec{p}, \vec{q}, 0)=\delta^{(2)}(\vec{p}-\vec{q}) /(2 \pi)$ and $\bar{\alpha}_{s}=\alpha_{s} N_{c} / \pi$. We start with the study of an observable similar to the usual Mueller-Navelet case such that we integrate over the azimuthal angles of the two central jets and over the difference in azimuthal angle between the two forward jets, $\Delta \theta=\vartheta_{A}-\vartheta_{B}-\pi$, considering so the mean value of the cosine

$$
\left\langle\cos \left(M\left(\vartheta_{A}-\vartheta_{B}-\pi\right)\right)\right\rangle=\frac{\int_{0}^{2 \pi} d \Delta \theta \cos (M \Delta \theta) \int_{0}^{2 \pi} d \vartheta_{1} \int_{0}^{2 \pi} d \vartheta_{2} \frac{d^{6} \sigma^{4-j e t}}{d k_{1} d y_{1} d \vartheta_{1} d k_{2} d \vartheta_{2} d y_{2}}}{\int_{0}^{2 \pi} d \Delta \theta \int_{0}^{2 \pi} d \vartheta_{1} \int_{0}^{2 \pi} d \vartheta_{2} \frac{d^{6} \sigma^{4-j e t}}{d k_{1} d y_{1} d \vartheta_{1} d k_{2} d \vartheta_{2} d y_{2}}} .
$$

Our next step now is to propose new observables, different from those characteristic of the Mueller-Navelet case though still related to azimuthal angle projections. We thus define

$$
\begin{aligned}
\mathscr{C}_{M N L}= & \int_{0}^{2 \pi} d \vartheta_{A} \int_{0}^{2 \pi} d \vartheta_{B} \int_{0}^{2 \pi} d \vartheta_{1} \int_{0}^{2 \pi} d \vartheta_{2} \cos \left(M\left(\vartheta_{A}-\vartheta_{1}-\pi\right)\right) \\
& \cos \left(N\left(\vartheta_{1}-\vartheta_{2}-\pi\right)\right) \cos \left(L\left(\vartheta_{2}-\vartheta_{B}-\pi\right)\right) \frac{d^{6} \sigma^{4-\mathrm{jet}}\left(\vec{k}_{A}, \vec{k}_{B}, Y_{A}-Y_{B}\right)}{d k_{1} d y_{1} d \vartheta_{1} d k_{2} d \vartheta_{2} d y_{2}}
\end{aligned}
$$

In order to improve the perturbative stability of our predictions (see [26] for a related discussion) it is convenient to remove the contribution from the zero conformal spin (which corresponds to the index $n=0$ in Eq. (2.3)) by defining the ratios

$$
\mathscr{R}_{P Q R}^{M N L}=\frac{\left\langle\cos \left(M\left(\vartheta_{A}-\vartheta_{1}-\pi\right)\right) \cos \left(N\left(\vartheta_{1}-\vartheta_{2}-\pi\right)\right) \cos \left(L\left(\vartheta_{2}-\vartheta_{B}-\pi\right)\right)\right\rangle}{\left\langle\cos \left(P\left(\vartheta_{A}-\vartheta_{1}-\pi\right)\right) \cos \left(Q\left(\vartheta_{1}-\vartheta_{2}-\pi\right)\right) \cos \left(R\left(\vartheta_{2}-\vartheta_{B}-\pi\right)\right)\right\rangle}
$$

with integer $M, N, L, P, Q, R>0$.

We present now a numerical analysis done in two characteristic configurations for the transverse momenta of the forward jets, namely $k_{A} \sim k_{B}$ and $k_{A}<k_{B}$ (or equivalently $k_{A}>k_{B}$ ), by fixing them to $\left(k_{A}, k_{B}\right)=(40,50)$ and to $\left(k_{A}, k_{B}\right)=(30,60) \mathrm{GeV}$. We also fix the rapidities of the four tagged jets to the values $Y_{A}=9, y_{1}=6, Y_{2}=3$, and $Y_{B}=0$ whereas the two inner jets can have transverse momenta in the range $20<k_{1,2}<80 \mathrm{GeV}$.

In Fig. 1 we show the behaviour for the normalized coefficients $\mathscr{C}_{121}$ and $\mathscr{C}_{211}$ after they are divided by their respective maximum. We find that the distributions are quite similar for the two configurations here chosen $\left(\left(k_{A}, k_{B}\right)=(40,50),(30,60) \mathrm{GeV}\right)$. Since these coefficients change sign in the $k_{1,2}$ range studied, it is clear that for the associated ratios $\mathscr{R}_{P Q R}^{M N L}$ there will be some lines of singularities. We present the behaviour of $\mathscr{R}_{122}^{111}$ and $\mathscr{R}_{211}^{222}$ in Fig. 2. In this case the configurations $\left(k_{A}, k_{B}\right)=(40,50),(30,60) \mathrm{GeV}$ behave quite differently. This is due to the variation of the position of the zeroes of those coefficients $\mathscr{C}_{M N L}$ chosen as denominators in these quantities. It would be very interesting to test if these singularity lines are present in any form in the LHC experimental data. In general, we notice a very weak dependence on variations of the rapidities $y_{1,2}$ of the more central jets for all the observables here presented.

We have used both FORTRAN and MATHEMATICA for the numerical computation of the ratios $\mathscr{R}_{P Q R}^{M N L}$. We made extensive use of the integration routine Vegas [27] as implemented in the Cuba library [28, 29]. Furthermore, we used the Quadpack library [30] and a slightly modified version of the Psi [31] routine.

It will be very important to compare against the BFKL Monte Carlo code BFKLex [32, 33, $34,35,36,37,38]$ as well as to calculate the same quantities with other, more conventional, approaches $[39,40,41]$ in order to gauge if they differ from our results. This includes those analysis where the four-jet predictions stem from two independent gluon ladders [42, 43, 44, 45]. 

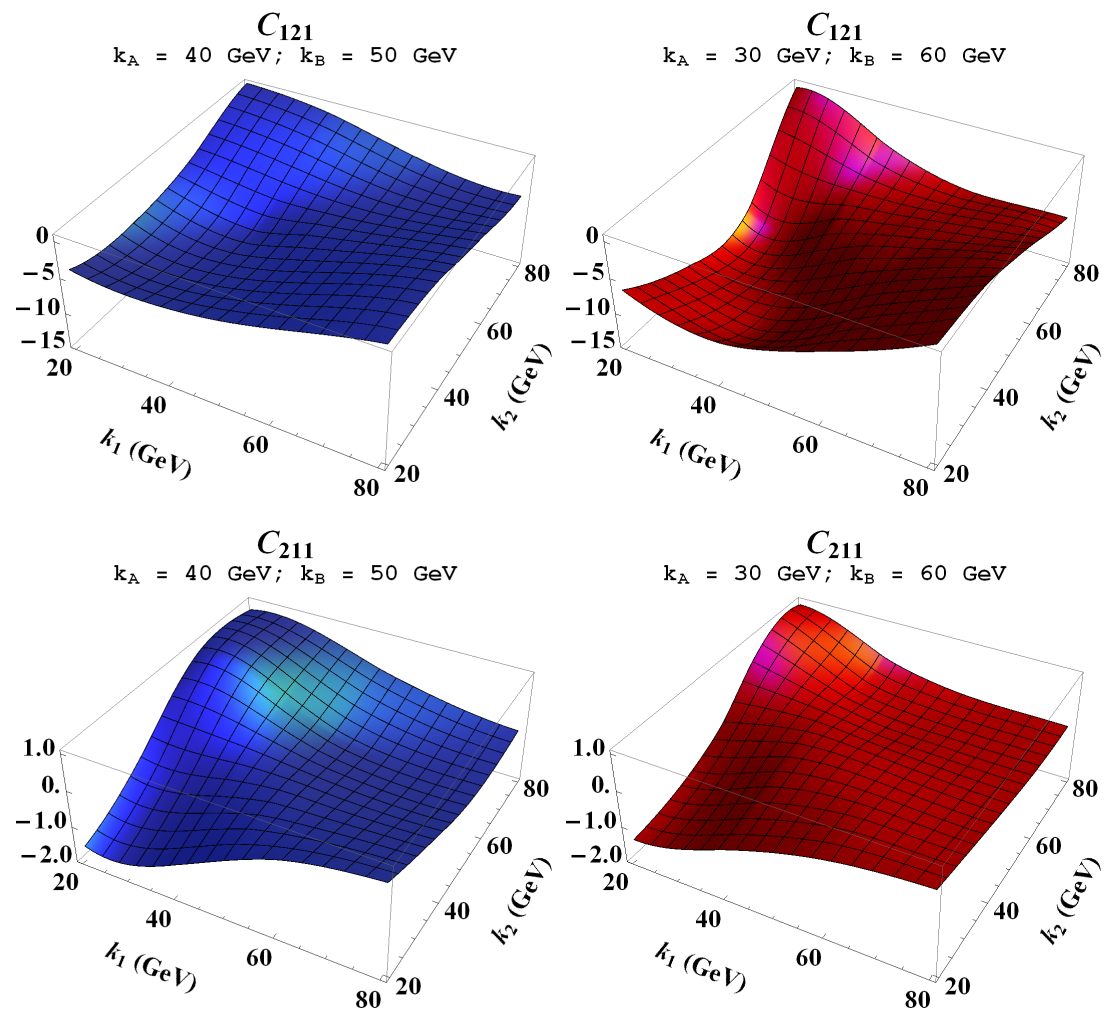

Figure 1: $k_{1,2}$-dependence of the normalized $\mathscr{C}_{121}$ and $\mathscr{C}_{211}$ for the two selected cases of forward jet transverse momenta $k_{A}$ and $k_{B}$.

\section{Conclusions \& Outlook}

We have considered ratios of correlation functions of products of azimuthal angle difference cosines in order to study four-jet production at hadron colliders. A single gluon ladder approach, with inclusive production of two forward/backward and two further, more central, tagged jets has been used. The dependence on the transverse momenta and rapidities of the two central jets represents a clear signal of BFKL dynamics. We continued and extended this study in [46] by giving more realistic predicions on the hadronc level through the introduction of parton distribution functions. For future works, more accurate analyses are needed: higher order effects and study of different configurations for the rapidity range of the two central jets. It is also interesting to calculate our proposed observables using other approaches not based on the BFKL approach and to test how they can differ from our predictions. In view of all these considerations, we encourage experimental collaborations to study these observables in the next LHC analyses.

\section{Acknowledgments}

G.C. acknowledges support from the MICINN, Spain, under contract FPA2013-44773-P. A.S.V. acknowledges support from Spanish Government (MICINN (FPA2015-65480-P)) and, together with F.C. and F.G.C., to the Spanish MINECO Centro de Excelencia Severo Ochoa Programme 


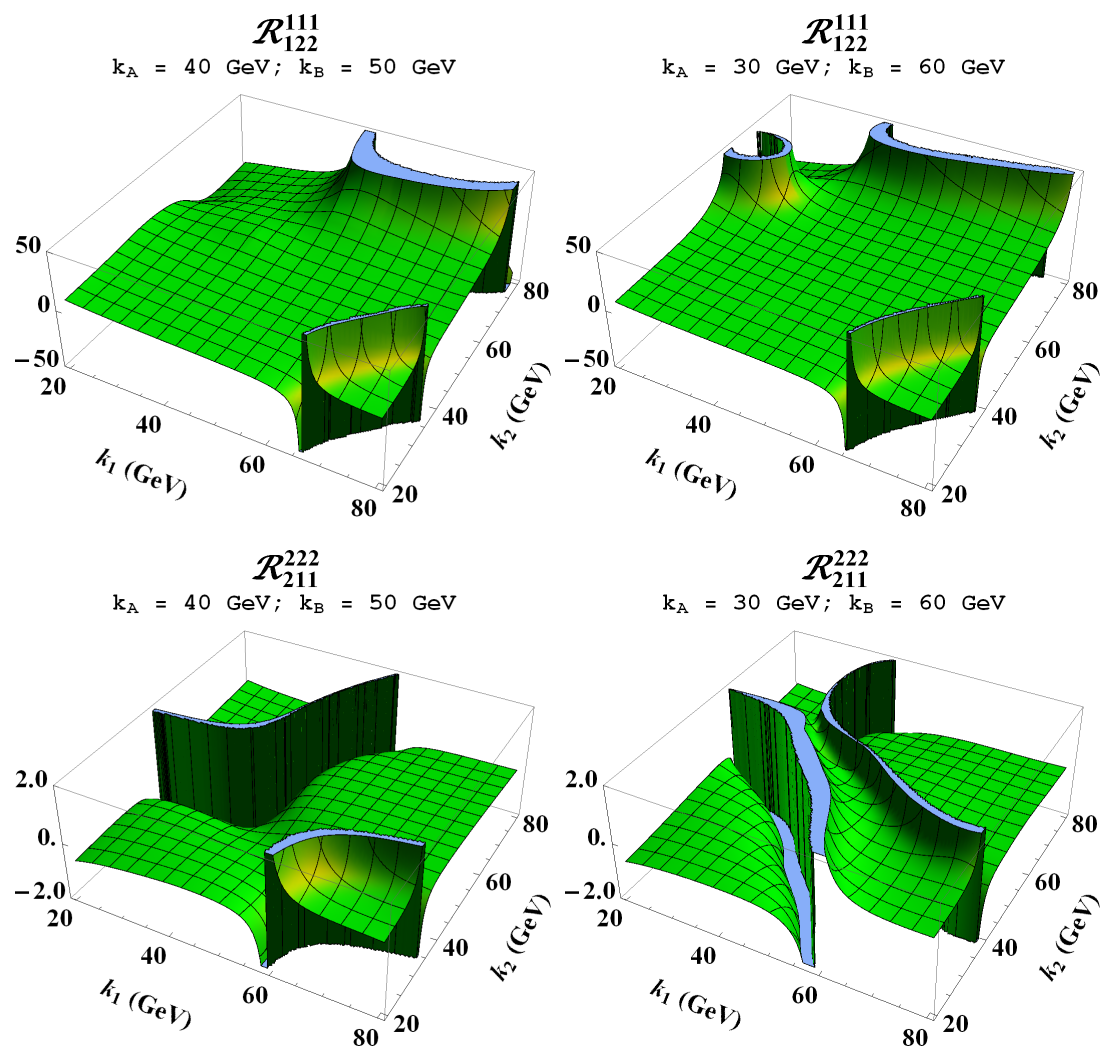

Figure 2: $k_{1,2}$-dependence of $\mathscr{R}_{122}^{111}$ and $\mathscr{R}_{211}^{222}$ for the two selected cases of forward/backward jets transverse momenta $k_{A}$ and $k_{B}$.

(SEV-2012-0249). F.G.C. thanks the Instituto de Fisica Teorica (IFT UAM-CSIC) in Madrid for warm hospitality.

\section{References}

[1] L. N. Lipatov, Sov. Phys. JETP 63 (1986) 904 [Zh. Eksp. Teor. Fiz. 90 (1986) 1536].

[2] I. I. Balitsky and L. N. Lipatov, Sov. J. Nucl. Phys. 28 (1978) 822 [Yad. Fiz. 28 (1978) 1597].

[3] E. A. Kuraev, L. N. Lipatov and V. S. Fadin, Sov. Phys. JETP 45 (1977) 199 [Zh. Eksp. Teor. Fiz. 72 (1977) 377].

[4] E. A. Kuraev, L. N. Lipatov and V. S. Fadin, Sov. Phys. JETP 44 (1976) 443 [Zh. Eksp. Teor. Fiz. 71 (1976) 840] [Erratum-ibid. 45 (1977) 199].

[5] L. N. Lipatov, Sov. J. Nucl. Phys. 23 (1976) 338 [Yad. Fiz. 23 (1976) 642].

[6] V. S. Fadin, E. A. Kuraev and L. N. Lipatov, Phys. Lett. B 60 (1975) 50.

[7] V. S. Fadin and L. N. Lipatov, Phys. Lett. B 429 (1998) 127 [hep-ph/9802290].

[8] M. Ciafaloni and G. Camici, Phys. Lett. B 430 (1998) 349 [hep-ph/9803389].

[9] M. Hentschinski, A. Sabio Vera and C. Salas, Phys. Rev. Lett. 110 (2013) 041601 [arXiv:1209.1353 [hep-ph]].

[10] M. Hentschinski, A. Sabio Vera and C. Salas, Phys. Rev. D 87 (2013) 076005 [arXiv:1301.5283 [hep-ph]].

[11] A. H. Mueller and H. Navelet, Nucl. Phys. B 282 (1987) 727.

[12] A. Sabio Vera, Nucl. Phys. B 746 (2006) 1 [hep-ph/0602250]. 
[13] A. Sabio Vera and F. Schwennsen, Nucl. Phys. B 776 (2007) 170 [hep-ph/0702158 [HEP-PH]].

[14] B. Ducloue, L. Szymanowski and S. Wallon, Phys. Rev. Lett. 112 (2014) 082003 [arXiv:1309.3229 [hep-ph]].

[15] F. Caporale, D. Y. Ivanov, B. Murdaca and A. Papa, Eur. Phys. J. C 74 (2014) 3084 [arXiv:1407.8431 [hep-ph]].

[16] F. G. Celiberto, D. Y. Ivanov, B. Murdaca and A. Papa, Eur. Phys. J. C 75 (2015) no.6, 292 [arXiv:1504.08233 [hep-ph]].

[17] F. G. Celiberto, D. Y. Ivanov, B. Murdaca and A. Papa, Eur. Phys. J. C 76 (2016) no.4, 224 [arXiv:1601.07847 [hep-ph]].

[18] R. Ciesielski, arXiv:1409.5473 [hep-ex].

[19] M. Angioni, G. Chachamis, J. D. Madrigal and A. Sabio Vera, Phys. Rev. Lett. 107, 191601 (2011) [arXiv:1106.6172 [hep-th]].

[20] G. Chachamis, arXiv:1512.04430 [hep-ph].

[21] F. Caporale, G. Chachamis, B. Murdaca and A. Sabio Vera, Phys. Rev. Lett. 116 (2016) no.1, 012001 [arXiv:1508.07711 [hep-ph]].

[22] F. Caporale, F. G. Celiberto, G. Chachamis, D. G. Gomez and A. Sabio Vera, arXiv:1603.07785 [hep-ph].

[23] D. Y. Ivanov and A. Papa, JHEP 1207 (2012) 045 [arXiv:1205.6068 [hep-ph]].

[24] F. G. Celiberto, D. Y. Ivanov, B. Murdaca and A. Papa, arXiv:1604.08013 [hep-ph].

[25] F. Caporale, F. G. Celiberto, G. Chachamis and A. Sabio Vera, Eur. Phys. J. C 76 (2016) no.3, 165 [arXiv:1512.03364 [hep-ph]].

[26] F. Caporale, B. Murdaca, A. Sabio Vera and C. Salas, Nucl. Phys. B 875 (2013) 134 [arXiv:1305.4620 [hep-ph]].

[27] G.P. Lepage, J. Comput. Phys. 27 (1978) 192.

[28] T. Hahn, Comput. Phys. Commun. 168 (2005) 78 [arXiv:1408.6373 [hep-ph]].

[29] T. Hahn, J. Phys. Conf. Ser. 608 (2015) 1 [arXiv:hep-ph/0404043].

[30] R. Piessens, E. De Doncker-Kapenga and C. W. Überhuber, Springer, ISBN: 3-540-12553-1, 1983.

[31] W. J. Cody, A. J. Strecok and H. C. Thacher, Math. Comput. 27 (1973) 121.

[32] G. Chachamis and A. Sabio Vera, PoS DIS 2013 (2013) 167 [arXiv:1307.7750].

[33] F. Caporale, G. Chachamis, J. D. Madrigal, B. Murdaca and A. Sabio Vera, Phys. Lett. B 724 (2013) 127 [arXiv:1305.1474 [hep-th]].

[34] G. Chachamis, A. Sabio Vera and C. Salas, Phys. Rev. D 87 (2013) 1, 016007 [arXiv:1211.6332 [hep-ph]].

[35] G. Chachamis and A. Sabio Vera, Phys. Lett. B 717 (2012) 458 [arXiv:1206.3140 [hep-th]].

[36] G. Chachamis and A. Sabio Vera, Phys. Lett. B 709 (2012) 301 [arXiv:1112.4162 [hep-th]].

[37] G. Chachamis, M. Deak, A. Sabio Vera and P. Stephens, Nucl. Phys. B 849 (2011) 28 [arXiv:1102.1890 [hep-ph]].

[38] G. Chachamis and A. Sabio Vera, arXiv:1511.03548 [hep-ph].

[39] M. Bury and A. van Hameren, Comput. Phys. Commun. 196, 592 (2015) [arXiv:1503.08612 [hep-ph]].

[40] M. A. Nefedov, V. A. Saleev and A. V. Shipilova, Phys. Rev. D 87, no. 9, 094030 (2013) [arXiv:1304.3549 [hep-ph]].

[41] A. van Hameren, P. Kotko and K. Kutak, JHEP 1212, 029 (2012) [arXiv:1207.3332 [hep-ph]].

[42] R. Maciula and A. Szczurek, Phys. Lett. B 749 (2015) 57 [arXiv:1503.08022 [hep-ph]].

[43] R. Maciula and A. Szczurek, Phys. Rev. D 90, no. 1, 014022 (2014) [arXiv:1403.2595 [hep-ph]].

[44] K. Kutak, R. Maciula, M. Serino, A. Szczurek and A. van Hameren, JHEP 1604 (2016) 175 [arXiv:1602.06814 [hep-ph]].

[45] K. Kutak, R. Maciula, M. Serino, A. Szczurek and A. van Hameren, arXiv:1605.08240 [hep-ph].

[46] F. Caporale, F. G. Celiberto, G. Chachamis, D. G. Gomez and A. Sabio Vera, arXiv:1606.00574 [hep-ph]. 